\title{
Damping rates of the atomic velocity in Sisyphus cooling
}

\author{
Laurent Sanchez-Palencia, Michele Schiavoni, François-Régis Carminati, Ferruccio Renzoni and Gilbert Grynberg \\ Laboratoire Kastler Brossel, Département de Physique de l'Ecole Normale Supérieure, \\ 24, rue Lhomond, 75231, Paris Cedex 05, France
}

(October 29, 2018)

\begin{abstract}
We present a theoretical and experimental study of the damping process of the atomic velocity in Sisyphus cooling. The relaxation rates of the atomic kinetic temperature are determined for a 3D lin $\perp$ lin optical lattice. We find that the damping rates of the atomic temperature depend linearly on the optical pumping rate, for a given depth of the potential wells. This is at variance with the behavior of the friction coefficient as calculated from the spatial diffusion coefficients within a model of Brownian motion. The origin of this different behavior is identified by distinguishing the role of the trapped and traveling atoms.
\end{abstract}

\section{INTRODUCTION}

The last decades have witnessed an impressive progress in laser cooling techniques, and nowdays it is possible to prepare and manipulate very cold and dense atomic samples [1,2]. Sisyphus cooling [3] represents a milestone in the history of laser cooling, particularly because this new cooling process demonstrated that the Doppler limit could be broken [4]. Furthermore, it is an important peculiarity of the Sisyphus process that the cooling mechanism not only leads to very low temperatures, but also to the strong localization of the atoms near the minima of the optical potential associated to the dipole force. Using appropriate laser configurations, it is then possible to design multidimensional modulations of the optical potential such that the atoms result spatially ordered in periodic arrays whose periodicity is of the order of the laser wavelength, the so-called optical lattices [5]. The possibility to prepare atoms in spatially ordered structures turned out to be essential for several studies in nonlinear dynamics [6-8].

Since the early works [3], Sisyphus cooling has been extensively studied, both experimentally and theoretically, in monodimensional as well as multidimensional optical lattices. The dependence of the steady-state kinetic temperature on the lattice parameters has been carefully examined [9-11]. It is by now a well established result that the temperature scales as the depth of the optical potential [9]. More recently, it has been shown that in a $3 \mathrm{D}$ lin $\perp$ lin configuration (see below) the temperature is on one hand independent of the lattice constant $[12,13]$ and on the other hand anisotropic $[13,14]$. Much less attention was payed to the study of the time scale of the damping of the atomic velocity in an optical lattice. So far, only indirect measurements of the cooling rate of atoms have been presented [15]. In that work, the cooling rates were inferred from the atomic localization rates measured by using Bragg scattering as a sensitive probe.

In this paper we report a theoretical and experimental study of the damping process of the atomic velocity in Sisyphus cooling. More precisely, we measure, both in the experiments and in the numerical simulations, the width of the atomic velocity distribution (which is proportional to the square root of the kinetic energy of the atomic sample) for different lattice durations within the thermalization phase. It has thus been possible to monitor the damping of the kinetic energy in a direct way. We show that the kinetic energy decreases exponentially during this phase and we determine the corresponding damping rate $\Gamma_{T}$ as a function of the lattice parameters (detuning from atomic resonance and intensity of the laser beams). We find that $\Gamma_{T}$ is proportional to the optical pumping rate and is strongly anisotropic. We also compare the cooling rate $\Gamma_{T}$ to the friction coefficient $\alpha$ as deduced from the spatial diffusion coefficients by using the Einstein relation for Brownian motion. We find that these two quantities show completely different dependencies on the lattice parameters. The origin of this different behavior is identified by distinguishing the role of the trapped and traveling atoms.

The present investigation is motivated by a parallel study of probe-transmission spectroscopy of atoms cooled in a dissipative optical lattice $[16,17]$. The properties of the optical lattice are studied by monitoring the transmission of a weak probe field through the atomic sample as a function of the relative detuning between the probe and the lattice fields. The knowledge of the relaxation rates of the different atomic observables (atomic density, atomic velocity, magnetization, etc) is necessary to understand the physical origin of the Rayleigh resonance that appears at the center of the probe transmission spectra. It is well established that the Rayleigh resonance originates from the scattering of photons from the lattice beams on the grating of an atomic observable induced by the pattern of interference between the probe and the lattice beams, and that the width of the Rayleigh line is given by the relaxation rate of 
this observable [18]. However which atomic observable determines the Rayleigh line in an optical lattice is still an open question [17]. Hence, the knowledge of the relaxation rates of the different atomic observables should allow, through comparison with the width of the Rayleigh line, the identification of the observable leading to the Rayleigh resonance.

\section{EXPERIMENTAL SET-UP}

In our experiment ${ }^{85} \mathrm{Rb}$ atoms are cooled and trapped in a 3D dissipative optical lattice red detuned with respect to the $F_{g}=3 \rightarrow F_{e}=4 \mathrm{D}_{2}$ line transition. The procedure to load the atoms in the optical lattice is the standard one used in previous experiments [12]: the rubidium atoms are first cooled and trapped in a magneto-optical trap (MOT) for about $1 \mathrm{~s}$; the temperature of the atoms in the MOT is of the order of $200 \mu \mathrm{K}$. Then the MOT magnetic field and laser beams are turned off and the lattice beams are turned on by using acousto-optical modulators (AOM) with a fall-rise time shorter than $1 \mu \mathrm{s}$.

The three-dimensional periodic optical lattice is generated by the interference of four linearly polarized laser beams, arranged in the $3 \mathrm{D}$ lin $\perp$ lin configuration [5] (Fig. 1): two $y$-polarized beams propagate in the $(x O z)$ plane with a relative angle $2 \theta$, while two $x$-polarized beams propagate in the $(y O z)$ plane and form also an angle $2 \theta$. This arrangement results in a periodic modulation of the light polarization and light intensity, which produces a periodic modulation of the light shifts of the different atomic ground-state sublevels (optical potentials). The optical potential has minima located on a orthorombic lattice and associated with pure circular (alternatively $\sigma^{+}$and $\sigma^{-}$) polarization. The lattice constants, i.e. the distance (along a major axis) between two sites of equal circular polarization are $\lambda_{x, y}=\lambda / \sin \theta$ and $\lambda_{z}=\lambda /(2 \cos \theta)$, where $\lambda$ is the laser field wavelength.

This configuration is well known to be suited for Sisyphus cooling [5] for which the steady-state kinetic energy of the atomic sample results from the competition of cooling and heating processes. The optical pumping between the different sublevels of the atomic ground state combined with the spatial modulation of the light shifts leads to the cooling of atoms, while the heating is essentialy due to the statistical fluctuations of the dipole force [3]. For deep enough potential modulations, Sisyphus cooling leads to atomic kinetic energies lower than the potential depth so that the atoms get trapped at the minima of the optical potential wells, thus producing a periodic array of atoms.

The temperature of the atoms is measured with the method of recoil-induced resonances [19-22]. The atoms are released from the lattice by turning off the lattice beams and two new laser beams (the pump (c) and the probe (p) beams, see Fig. 1) are introduced. Beams $\mathrm{c}$ and $\mathrm{p}$ are detuned by $49 \mathrm{MHz}$ from atomic resonance and their intensities are of the order of $100 \mu \mathrm{W} / \mathrm{cm}^{2}$. They cross the atomic sample in the $(x O z)$ plane, and they are symmetrically displaced with respect to the $z$ - axis forming an angle of $2 \varphi=25^{\circ}$. The diamater of the pump beam is about $1 \mathrm{~cm}$, larger than the atomic cloud, while the probe beam is very thin (less than $500 \mu \mathrm{m}$ in diameter). The frequency of the probe beam is scanned around the pump frequency in $1 \mathrm{~ms}$. We notice that for these parameters of the pump and probe beams, the heating due to their absorption is negligible. The probe transmission is monitored as a function of the detuning $\delta$ between the pump and probe fields. For equal pump and probe polarizations, the ground states $|\alpha, p\rangle$ and $\left|\alpha, p^{\prime}\right\rangle$ with the same internal quantum number $\alpha$ but different atomic momenta are coupled by Raman transitions. The difference in population of these states determines the absorption or gain of the probe, and the probe transmission spectrum is thus proportional to the derivative of the atomic velocity distribution [20]. These distributions were found to fit well to Gaussian functions so that it is straightforward to calculate the mean kinetic energy $E_{K}$ in the $j$ - direction and to derive an atomic kinetic temperature

$$
E_{K_{j}}=\frac{k_{B} T_{j}}{2}
$$

from the width of the resonance in the probe transmission spectrum. The geometry of our pump and probe fields corresponds to measurement of the temperature in the $x$ - direction.

\section{EXPERIMENTAL RESULTS}

In the experiment, the duration of the optical lattice is carefully controlled and can be varied in steps of $10 \mu \mathrm{s}$. We measure the kinetic temperature of the atomic cloud as a function of the duration $t$ of the optical lattice for different lattice parameters (intensity per lattice beam $I_{L}$ and lattice detuning $\Delta$ from atomic resonance). We plot in Fig. 2 a typical variation of the measured kinetic temperature $T_{x}$ as a function of the time $t$ together with a fit of the data to a function of the form

$$
T_{x}(t)=T_{f_{x}}+\left(T_{i}-T_{f_{x}}\right) \exp \left(-\Gamma_{T_{x}} \cdot t\right) .
$$


The kinetic temperature decreases exponentially with the lattice duration $t$ towards a steady-state temperature, and the fit to Eq. (2) provides the steady-state temperature $T_{f_{x}}$ as well as the damping rate $\Gamma_{T_{x}}$ of the atomic temperature. From experimental data as those of Fig. 2, we determined $\Gamma_{T_{x}}$ and $T_{f_{x}}$ in a broad range of lattice parameters (intensity per lattice beam $I_{L}$ and lattice detuning $\Delta$ ). We found that the temperature shows a linear increase with $I_{L}$ and decreases against $\Delta$, in agreement with earlier studies. A more complete account of the dependence of the steady-state temperature on the lattice parameters has already been reported in Ref. [12] and will not be repeated here. The novel result of the present work consists instead in the determination of the damping rate of the temperature.

Experimental results for $\Gamma_{T_{x}}$ are reported in Fig. 3 as a function of the lattice beam intensity at fixed lattice detuning (top) and as a function of the optical pumping rate $\Gamma_{0}^{\prime} \propto I_{L} / \Delta^{2}$ at fixed light shift per beam $\Delta_{0}^{\prime} \propto I_{L} / \Delta$ (bottom). We find that $\Gamma_{T_{x}}$ ranges from a few $\mathrm{kHz}$ to a few tens of $\mathrm{kHz}$ for typical intensity and detuning of the lattice beams. More precisely, from the experimental data, we identify the following general behavior of the damping rate as a function of the lattice parameters: $\Gamma_{T_{x}}$ is an increasing function of the lattice beams intensity $I_{L}$, the dependence being linear within the experimental error, and a decreasing function of the lattice detuning $|\Delta|$. When plotted against the optical pumping rate, $\Gamma_{T_{x}}$ is found to be proportional to $\Gamma_{0}^{\prime}$, and almost independent of the light shift per beam. The observed behavior is in good agreement with the results of Raithel et al [15], and will be interpreted with the help of Monte-Carlo simulations.

\section{THEORETICAL ANALYSIS}

We analyze theoretically the damping process of the atomic velocity with the help of semi-classical Monte-Carlo simulations. Taking advantage of the symmetry between the $x-$ and $y$ - directions (see Fig. 1) we restricted the atomic dynamics in the $(x O z)$ plane. The calculations are made for a $J_{g}=1 / 2 \rightarrow J_{e}=3 / 2$ atomic transition, as customary done in numerical analysis of Sisyphus cooling. The numerical calculations in this simplified configuration are expected to give the same dependencies of the dynamical quantities on the lattice parameters as full 3D calculations, so they are useful to study the main feautures of the cooling dynamics. However, a direct quantitative comparison to the experimental results is not necessarily meaningful because the restriction of the dynamics to two dimensions may introduce scaling factors [13].

\section{A. Cooling rates}

We first determine the mean kinetic energies along the $x$ - and $z$-directions as functions of time in the thermalization phase. The mean kinetic energy is obtained by averaging over about 5000 trajectories. For both directions, the temperature shows an exponential decrease with the lattice duration. By fitting the temperature $T_{x}$ and $T_{z}$ with a function of the form (2), we determine the corresponding relaxation rates $\Gamma_{T_{j}}$ in the $x-$ and $z-$ directions, with results as in Fig. 4.

We notice that the damping rates for the two orthogonal directions are very different, with $\Gamma_{T_{x}}$ typically about one order of magnitude smaller than $\Gamma_{T_{z}}$. This property certainly relates to the strong anisotropies of the optical potential and of the optical pumping rates in the $3 \mathrm{D}$ lin $\perp$ lin lattice. Previous work already pointed out anisotropic temperatures $[13,14]$ and anisotropic spatial diffusion coefficients $[12,13]$ for a $3 \mathrm{D}$-lin $\perp$ lin lattice.

The graphs of the left-hand column of Fig. 4 report the results for the damping rates $\Gamma_{T_{j}}$ as functions of the intensity per lattice beam $I_{L}$ for different values of the lattice detuning $\Delta$ and confirm the general behavior observed in the experiment: $\Gamma_{T_{j}}$ is an increasing function of the lattice intensity, and a decreasing function of the lattice detuning.

On the right-hand column of Fig. 4 the numerical results for the damping rates are reported as functions of the optical pumping rate per lattice beam $\Gamma_{0}^{\prime}$ for fixed values of the light shift per beam $\Delta_{0}^{\prime}$, i.e. for fixed values of the depth of the potential wells. This allows a more transparent interpretation of our results. Indeed, the damping rates are found to be proportional to the optical pumping rate in a large range of light shifts per lattice beam within a numerical error of about $10 \%$. This is in qualitative agreement with our experimental observations, and also with the indirect measurements of the cooling rates of Raithel et al [15].

\section{B. Friction coefficients}

It is interesting to compare the results obtained for the damping rate of the kinetic energy with the well known friction coefficients for Sisyphus cooling [3]. Such a coefficient $\alpha$ is introduced in the low-velocity limit where the mean cooling force has the form $F=-\alpha v$. We determined the friction coefficients $\alpha_{x}$ and $\alpha_{z}$ for the two orthogonal 
directions. A possible way to calculate the friction coefficients is to introduce an arbitrary constant force $F_{j}$ along the direction $j$. The mean friction force compensates this force, with the atomic cloud set into a motion along $j$ at a constant mean velocity $\left\langle v_{j}\right\rangle=F_{j} / \alpha_{j}$. The friction coefficient $\alpha_{j}$ is then easily derived by calculating the velocity $\left\langle v_{j}\right\rangle$ for various $F_{j}$. A different approach to the calculation of the friction coefficients is based on the assumption that the atomic dynamics can be described as a Brownian motion. Under this assumption, the friction coefficients can be derived from the steady-state kinetic temperature $T_{j}$ and the spatial diffusion coefficient $D_{s_{j}}$ in the $j$-direction by using the Einstein relation for Brownian motion $\alpha_{j}=k_{B} T_{j} / D_{s_{j}}$. In previous work [13], these two approaches have been compared and shown to lead to the same values for the friction coefficients. Also for the present choice of interaction parameters, we found that the two methods lead to values of $\alpha_{j}$ in excellent agreement [23]. These values are shown in Fig. 5 as functions of $\Gamma_{0}^{\prime}$ for different values of the light shift per beam $\Delta_{0}^{\prime}$. We notice that also for the friction coefficients there is a strong anisotropy, with $\alpha_{x}$ about 5 times smaller than $\alpha_{z}$. The results for the friction coefficients have to be compared with the values of $\Gamma_{T_{x}}$ and $\Gamma_{T_{z}}$ shown in Fig. 4 . The values $\alpha_{j} / M$ are about one order of magnitude larger than those for $\Gamma_{T_{j}}$. Furthermore, the two quantities do not show the same dependence on the lattice parameters. In fact, $\alpha_{j}$ weakly depends on $\Gamma_{0}^{\prime}$ but strongly depends on $\Delta_{0}^{\prime}$ in the range of parameters considered here. This behaviour is completely different from the one for $\Gamma_{T_{j}}$ in the same range of lattice parameters.

\section{Discussion}

The very different dependence on the lattice parameters shown by the damping rates of the kinetic energy and the friction coefficients is rather surprising. In fact, the description of the atomic dynamics as a Brownian motion suggests that the friction coefficient in a given direction, rescaled by the atomic mass $M$, is simply the half of the damping rate of the kinetic energy in the same direction. The different behavior shown by these two quantities can be explained by distinguishing the role of the trapped and traveling atoms. In fact both in the thermalization phase and at thermal equilibrium there are high energy atoms (h-e) that travel over the lattice sites and low energy atoms (l-e) that are trapped in the potential wells. As discussed in detail in Ref. [13], the high energy atoms experience Sisyphus cooling, undergo a Brownian motion, and their dynamics can be characterized by a friction coefficient $\alpha_{j}$. Since these atoms undergo a Brownian motion one may expect

$$
\left(\frac{\alpha_{j}}{M}\right)_{h-e}=\left(\frac{\Gamma_{T_{j}}}{2}\right)_{h-e}
$$

On the other hand, low-energy atoms are trapped in the potential wells and do not undergo a Brownian motion. Therefore they show a very large (ideally divergent) friction coefficient. In fact, the application of a weak force $F_{j}$ does not set them into a uniform motion, but only changes their center of oscillation in the wells. Therefore for these atoms the friction coefficient $\alpha_{j}=F_{j} /\left\langle v_{j}\right\rangle$ is divergent. Furthermore, trapped atoms do not loose kinetic energy by Sisyphus cooling and therefore they are characterized by a cooling rate $\Gamma_{T}$ ideally equal to zero. It follows that for the low-energy trapped atoms

$$
\left(\frac{\alpha_{j}}{M}\right)_{l-e} \gg\left(\frac{\Gamma_{T_{j}}}{2}\right)_{l-e}
$$

It is then clear that the presence of trapped atoms invalidates the simple relation $\alpha_{j} / M=\Gamma_{T_{j}} / 2$ and lead to a cooling rate $\Gamma_{T_{j}}$ smaller than $2 \alpha_{j} / M$, as observed in our numerical simulations.

\section{CONCLUSIONS}

In summary, we have determined experimentally the damping rates of the atomic kinetic energy (kinetic temperature) in Sisyphus cooling, and have examined their general dependencies on the lattice parameters. We have also made numerical semi-classical Monte-Carlo simulations. We have shown that the damping rates of the atomic kinetic temperature depend linearly on the optical pumping rate and are strongly anisotropic. We have also determined the friction coefficients from the atomic spatial diffusion coefficinets and we have shown that they weakly depend on the optical pumping rate but strongly depend on the potential depth. The very different behavior of the damping rate of the kinetic temperature $\Gamma_{T_{j}}$ and of the friction coefficient $\alpha_{j}$ has been explained by distinguishing the role of the trapped and traveling atoms.

The presented results for the damping rates of the atomic kinetic temperature in Sisyphus cooling are useful for the analysis of the Rayleigh resonance detected in probe-transmission spectroscopy of atoms cooled in dissipative 
optical lattices $[16,17]$. Indeed, the knowledge of the damping rates of the different atomic observables excited in the optical process is necessary to determine the physical origin of the resonance. Preliminary measurements for the probe transmission show that the width of the Rayleigh resonance is of the same order of magnitude of the value for $\Gamma_{T_{j}}$ presented in this work. This suggests that the atomic velocity is one of the observables which, excited in the optical

process determined by the probe and lattice fields, contribute to the Rayleigh resonance. Further work is however necessary for a definitive conclusion.

\section{ACKNOWLEDGMENTS}

Laboratoire Kastler Brossel is an "unité mixte de recherche de l'Ecole Normale Supérieure et de l'Université Pierre et Marie Curie associée au Centre National de la Recherche Scientifique (CNRS)". 
[1] S. Chu, "Nobel lectures: The manipulation of neutral particles", Rev. Mod. Phys. 70, 685 (1998); C. Cohen-Tannoudji, "Nobel lectures: manipulating atoms with photons", ibid 70, 707 (1998); W.D. Phillips, "Nobel lectures: laser cooling and trapping of neutral atoms", ibid 70, 721 (1998).

[2] H.J. Metcalf and P. van der Straten, Laser cooling and trapping (Springer-Verlag, Berlin, 1999).

[3] J. Dalibard and C. Cohen-Tannoudji, "Laser cooling below the Doppler limit by polarization gradients: simple theoretical models", J. Opt. Soc. Am. B 6, 2023 (1989); P.J. Ungar, D.S. Weiss, E. Riis and S. Chu, "Optical molasses and multilevel atoms: theory", ibid 6, 2058 (1989).

[4] P. D. Lett, R. N. Watts, C. I. Westbrook, W. D. Phillips, P. L. Gould and H. J. Metcalf, "Observation of atoms laser cooled below the Doppler limit", Phys. Rev. Lett. 61, 169 (1988).

[5] G. Grynberg and C. Mennerat-Robilliard, "Cold atoms in dissipative optical lattices", Phys. Rep. 355, 335 (2001).

[6] M.G. Raizen, "Quantum chaos with ultra-cold atoms" Comments At. Mol. Phys. 34, 321 (1999); M.G. Raizen, "Quantum chaos with ultra-cold atoms" Adv. At. Mol. Opt. Phys. 41, 43 (1999).

[7] L. Sanchez-Palencia, F.-R. Carminati, M. Schiavoni, F. Renzoni and G. Grynberg, "Brillouin propagation modes in optical lattices: interpretation in terms of nonconventional stochastic resonance", Phys. Rev. Lett. 88, 133903 (2002).

[8] M. Schiavoni, F.-R. Carminati, L. Sanchez-Palencia, F. Renzoni and G. Grynberg, "Stochastic resonance in periodic potentials: realization in a dissipative optical lattice", Europhys. Lett. 59, 493 (2002).

[9] M. Gatzke, G. Birkl, P.S. Jessen, A. Kastberg, S.L. Rolston, and W.D. Phillips, "Temperature and localization of atoms in three-dimensional optical lattices", Phys. Rev. A 55, R3987 (1997).

[10] J. Jersblad, H. Ellmann and A. Kastberg, "Experimental investigation of the limit of Sisyphus cooling", Phys. Rev. A 62, 051401(R) (2000).

[11] H. Ellmann, J. Jersblad and A. Kastberg, "Temperatures in 3D optical lattices influenced by neighbouring transitions", Eur. Phys. J. D 13, 379 (2001).

[12] F.-R. Carminati, M. Schiavoni, L. Sanchez-Palencia, F. Renzoni and G. Grynberg, "Temperature and spatial diffusion of atoms cooled in a 3D lin $\perp$ lin bright optical lattice", Eur. Phys. J. D 17, 249 (2001).

[13] L. Sanchez-Palencia, P. Horak and G. Grynberg, "Spatial diffusion in a periodic optical lattice: revisiting the Sisyphus effect", Eur. Phys. J. D 18, 353 (2002).

[14] J. Jersblad, H. Ellmann, L. Sanchez-Palencia and A. Kastberg, "Anisotropic velocity distributions in 3D dissipative optical lattices", Eur. Phys. J. D, in press.

[15] G. Raithel, G. Birkl, A. Kastberg, W.D. Phillips and S.L. Rolston, "Cooling and localization dynamics in optical lattices", Phys. Rev. Lett. 78, 630 (1997).

[16] F.-R. Carminati, M. Schiavoni, Y. Todorov, F. Renzoni and G. Grynberg, "Pump-probe spectroscopy of atoms cooled in a $3 \mathrm{D}$ lin $\perp$ lin optical lattice", Eur. Phys. J. D, in press.

[17] F.-R. Carminati, L. Sanchez-Palencia, M. Schiavoni, F. Renzoni and G. Grynberg, "Rayleigh scattering and atomic dynamics in optical lattices", to be published.

[18] J.-Y. Courtois and G. Grynberg, "Stimulated Rayleigh resonances and recoil-induced effect", Adv. At. Mol. Opt. Phys. 36, 87 (1996).

[19] J.-Y. Courtois, G. Grynberg, B. Lounis and P. Verkerk, "Recoil-induced resonances in cesium: an atomic analog to the free-electron laser", Phys. Rev. Lett. 72, 3017 (1994).

[20] D.R. Meacher, D. Boiron, H. Metcalf, C. Salomon and G. Grynberg, "Method for velocimetry of cold atoms", Phys. Rev. A 50, R1992 (1994).

[21] F. Chi, M. Partlow and H. Metcalf, "Precision velocimetry in a thermal atomic beam by stimulated optical Compton scattering", Phys. Rev. A 64, 043407 (2001).

[22] G. Di Domenico, G. Mileti and P. Thomann, "Pump-probe spectroscopy and velocimetry of cold atoms in a slow beam", Phys. Rev. A 64, 043408 (2001).

[23] We also verified that the same values for $\alpha_{j}$ are obtained by considering as initial atomic distribution an ensemble of atoms well localized in deep potential wells, and then reducing the depth of the wells to the wanted value for the measurement of $\alpha_{j}$. 


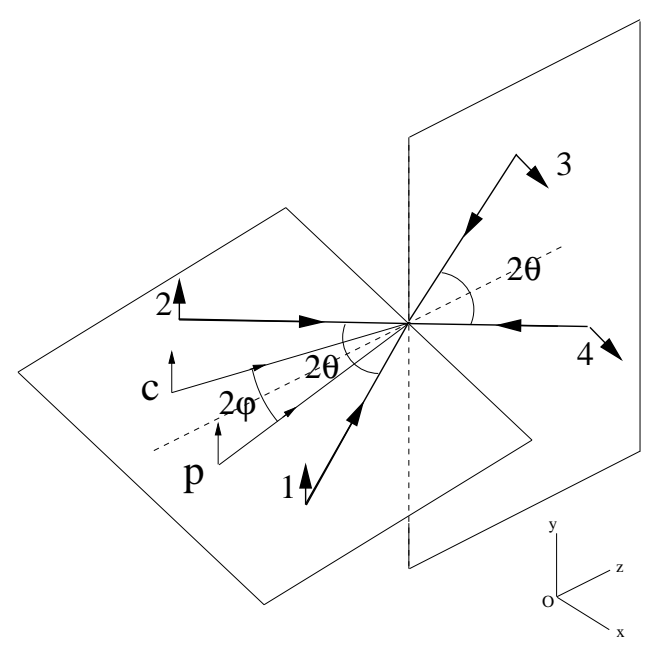

FIG. 1. Laser fields configuration for the $3 \mathrm{D}$-lin $\perp$ lin optical lattice. The beams $1-4$ generate the $3 \mathrm{D}$ periodic potential. For the measurement presented in this work, the angles between the lattice beams are kept fixed at $\theta=30^{\circ}$. Two additional laser beams (c and p), are introduced for the temperature measurement. They are both linearly polarized along the $y$-axis. The angle between beams c and $\mathrm{p}$ is $2 \varphi=25^{\circ}$.

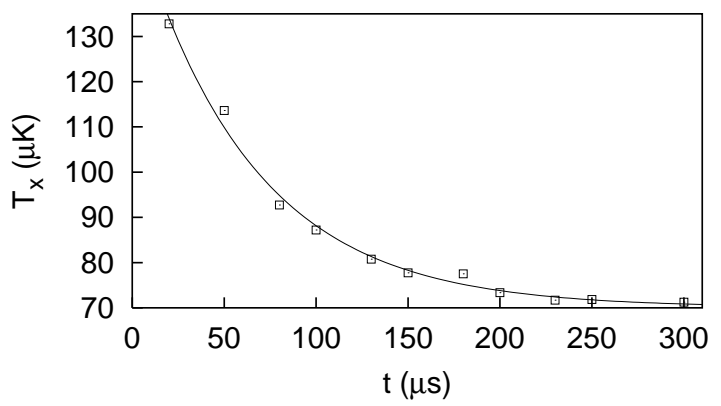

FIG. 2. Experimental results for the temperature $T_{x}$ in the $x$-direction of the atoms cooled in the optical lattice as a function of the duration $t$ of the lattice. The lattice detuning is $\Delta=48 \mathrm{MHz}$, the intensity per lattice beam $I_{L}=4.5 \mathrm{~mW} / \mathrm{cm}^{2}$. The solid line is the fit to the exponentially decreasing function Eq. (2). 

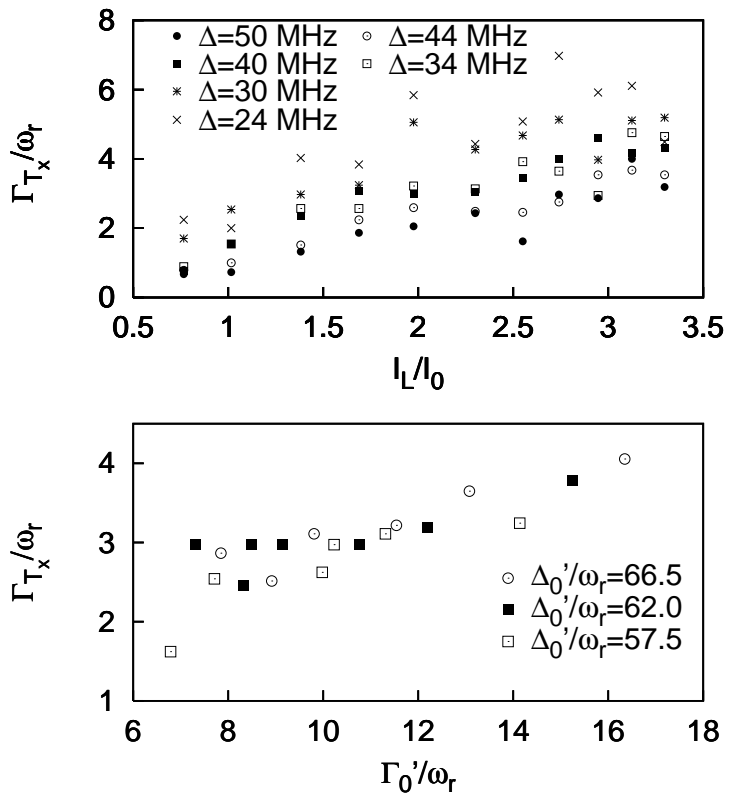

FIG. 3. Experimental results for the damping rate $\Gamma_{T_{x}}$ of the atomic temperature in the $x-$ direction. In the upper graph $\Gamma_{T_{x}}$ is plotted as a function of the intensity per lattice beam $I_{L}$, for different values of the lattice detuning $\Delta$. In the lower graph $\Gamma_{T_{x}}$ is plotted as a function of the optical pumping rate $\Gamma_{0}^{\prime}$, for different values of the light shift per beam $\Delta_{0}^{\prime}$. Here $\omega_{r}$ is the atomic recoil frequency, and $I_{0}$ the saturation intensity.
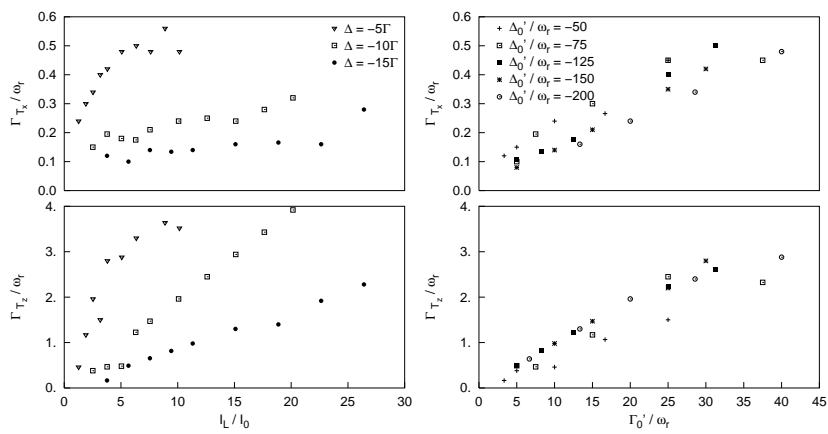

FIG. 4. Numerical results for the damping rates of the atomic temperature in the $x-$ and $z$ - directions. In the left hand column the damping rates are plotted as functions of the intensity per lattice beam $I_{L}$, for different values of the lattice detuning $\Delta$. In the right hand column, the same data are reported as functions of the optical pumping rate $\Gamma_{0}^{\prime}$, for different values of the light shift per beam $\Delta_{0}^{\prime}$. Here $\Gamma$ is the width of the excited state. 

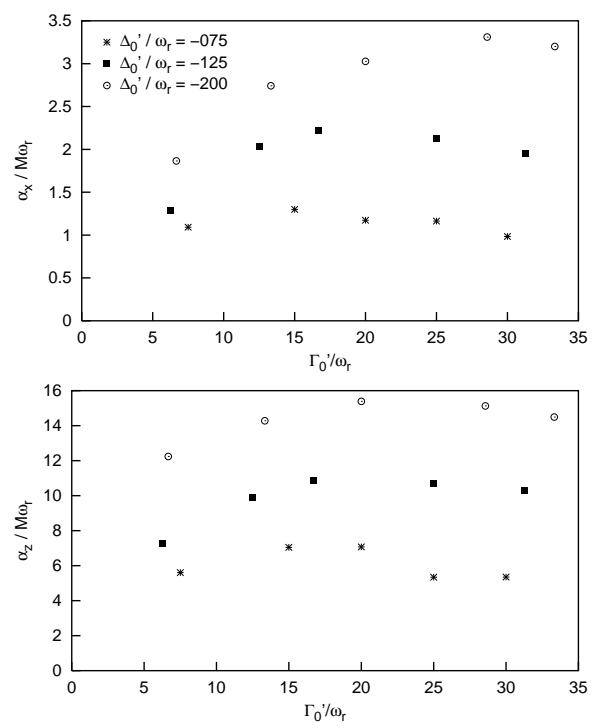

FIG. 5. Numerical results for the friction coefficients in the $x$ - and $z$-directions as functions of the optical pumping rate $\Gamma_{0}^{\prime}$ for various values of the light shift per beam $\Delta_{0}^{\prime}$. 